\title{
Posterior Reversible Encephalopathy Syndrome in a Case of Postoperative Spinal Extradural Haematoma: Case Report and Review of Literature
}

\author{
Chittur Viswanathan Gopalakrishnan, Vazhayil Vikas, Suresh Nair \\ Department of Neurosurgery, Sree Chitra Tirunal Institute for Medical Sciences and Technology, \\ Trivandrum, Kerala, India
}

A 14-year-old girl presented with progressive paraparesis and paresthesia of one-year duration. Magnetic resonance imaging revealed a T6 vertebral hemangioma with epidural compression on the spinal cord. Following angiography and embolization, she underwent dorsal laminectomy and excision of the soft tissue component compressing the cord. In the postoperative period she had rapid worsening of lower limb power and imaging demonstrated an epidural haematoma at the operative site. The patient was taken up for urgent re-exploration and evacuation of haematoma. Postoperatively the patient complained of visual failure, headache and had multiple episodes of seizures. An magnetic resonance imaging brain showed characteristic features of posterior reversible encephalopathy syndrome (PRES) and the patient improved gradually after control of hypertension. This is the first documented case of PRES following spinal cord compression in a patient without any known risk factors. We postulate the possible mechanism involved in its pathogenesis.

Key Words: Posterior reversible encephalopathy syndrome, Magnetic resonance imaging, Hematoma, epidural, spinal

\section{Introduction}

The posterior reversible encephalopathy syndrome (PRES) is a well-recognized cliniconeuroradiologic syndrome. Hinchey et al. [1] first described the syndrome in 1996. The common clinical features are headache, seizures, visual changes, altered mental status, and focal neurological signs. Magnetic resonance imaging (MRI) often reveals reversible, usually symmetric, hyperintense lesions on T2weighted scans, which involve the white matter in the posterior parietal and occipital areas of the brain, although gray matter structures may also be affected. It has now become clear that lesions are not necessarily in the parieto-occipital lobes and cerebellum but may also be found in the frontal lobes, temporal lobes and brainstem. PRES is usually a reversible condition if promptly recognized and appropriately treated. It is generally observed in the setting of secondary hypertension and following administration of immunosuppressive drugs. The most widely accepted hypotheses regarding its mechanism are the generation of vasogenic edema caused by failure of cerebral autoregulation and the production of cytotoxic edema due to ischemia. Here, we report a case with clinical and imaging findings consistent with PRES that occurred after spinal surgery without any of the common risk factors. PRES has not been reported earlier following spinal surgery. We also postulate the possible mechanism involved in the pathogenesis of PRES in this case.

Received Jul 20, 2010; 1st Revised Aug 30, 2010; Accepted Aug 31, 2010

Corresponding author: Chittur Viswanathan Gopalakrishnan, MD

Department of Neurosurgery, Sree Chitra Tirunal Institute for Medical Sciences and Technology,

Trivandrum, Kerala 695011, India

Tel: +91-471-2524647, Fax: +91-471-2446433, E-mail: doc_gopal@yahoo.com 


\section{Case Report}

A 14-year-old girl presented with back pain in the midthoracic region followed by rapidly progressive weakness and paresthesia in both lower limbs of 2 weeks duration.
Her past medical history was unremarkable. At the time of admission she was non-ambulant. On examination, she had grade 2 power in both lower limbs with mild hypertonia, brisk reflexes and extensor plantar response. She had blunting of pinprick and temperature sensations upto T10 level. MRI spine revealed a hypointense lesion on T1-weighted
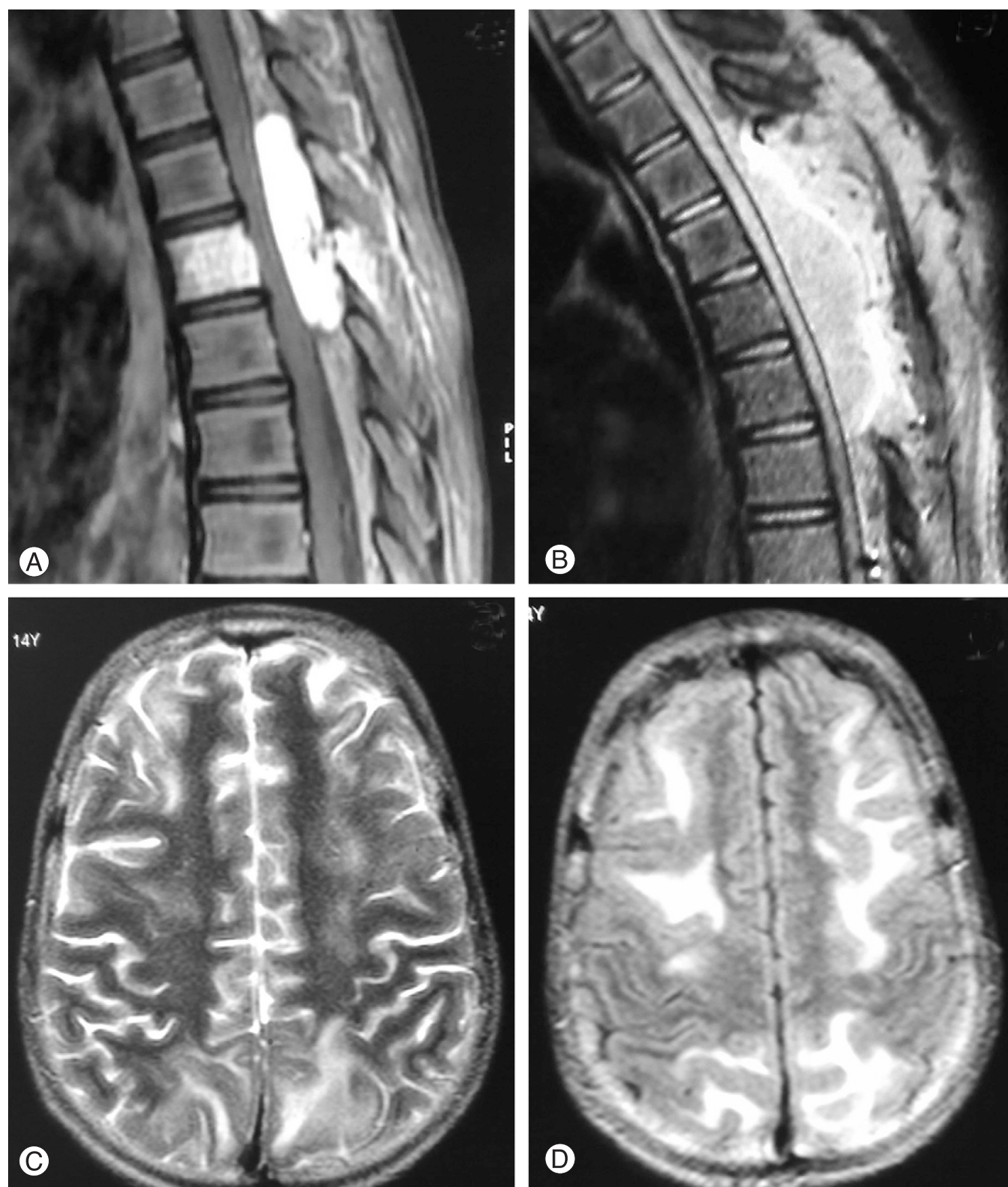

Fig. 1. Post contrast $\mathrm{T} 1$ weighted sagittal magneric resonance imaging (MRI) spine (A) shows a T6 haemangioma with a large extradural component compressing the cord. Postoperative T1 weighted sagittal MRI (B) demonstrates a haematoma at the operative site. Transverse T2-weighted (C) and fluid-attenuated inversion-recovery-weighted (D) MRI sequences show typical findings consistent with vasogenic oedema located in bilateral frontal and parietooccipital regions. 
image that was hyperintense on T2-weighted image, involving the T6 vertebral body with a significant epidural component causing spinal cord compression. The entire lesion was well enhancing on gadolinium administration (Fig. 1A). An imaging diagnosis of T6 haemangioma was made and the patient underwent angiography and embolization of the intercostals arteries supplying the lesion. Post embolization angiography showing significant reduction in tumour vascularity, but in view of the extradural component producing cord compromise the patient underwent a T4-T7 laminectomy and excision of the soft tissue lesion. There were no complications noted during or immediately after the procedure. Perioperative blood pressure (BP) recordings were normal. Histopathological examination of the lesion revealed closely packed, thin walled small vessels of capillary caliber lined by endothelial cells and arranged in a lobular pattern. The intervening stroma was composed of loose fibrocollagenous tissue with myxoid areas. These findings were consistent with a pathological diagnosis of haemangioma.

Immediate postoperative assessment of lower limb power was same as preoperative status. Three hours after surgery, on repeat assessment, it was noted that the patient's lower limb power had deteriorated significantly. An urgent MRI spine showed epidural haematoma at the operative site (Fig. 1B) and the patient was taken up for re-exploration and evacuation of the haematoma. She was induced with thiopentone and anaesthesia was maintained with sevoflurane. The operative procedure was uneventful and the patient was extubated. Twelve hours after extubation the patient complained of headache and bilateral visual loss though ophthalmologic evaluation was normal. Her sensorium gradually deteriorated and she had multiple episodes of generalized tonic-clonic seizures for which she had to be ventilated and started on barbiturate sedation. Her biochemical and haematological evaluation was normal. Electroencephalography showed few sharp waves over the left temporal area with moderate diffuse slow wave abnormality over left fronto-temporal area. MRI brain showed bilateral hyperintensities in $\mathrm{T} 2$ weighted and fluid-attenuated inversion-recovery sequences in the subcortical frontal and parieto-occipital regions (Fig. 1C and 1D). The lesions were non-enhancing on contrast administration and showed no associated changes on diffusion-weighted imaging (DWI). Concomitant magnetic resonance angiography was normal as well. Barbiturates were stopped after 4 days and she was gradually weaned off ventilator support. The patient remained confused for a few days and no more seizures were observed. Her visual acuity was normal at the time of discharge from the hospital. Her lower limb weakness remained the same as pre-operative state and she was started on aggressive physiotherapy.

\section{Discussion}

PRES was introduced in 1996 in order to describe a unique syndrome, seen mainly during hypertensive encephalopathy [1]. It has also been described as reversible posterior cerebral edema syndrome and posterior reversible leukoencephalopathy by different authors. The constellation of symptoms and signs described include headache, seizures, visual changes, altered mentation and occasionally focal neurologic signs. Visual disturbances range from blurred vision up to cortical blindness.

The pathophysiology of PRES is uncertain. Though commonly observed in patients suffering from acute hypertension, eclampsia and renal disease, it has also been reported in the clinical setting of hypocholesterolemia, hypomagnesaemia, hypercalcaemia, aluminum overload, high-dose methylprednisolone therapy, uremic encephalopathy, pheochromocytoma, systemic lupus erythematosus, Henoch-Schönlein purpura, acute hepatic failure, acute intermittent porphyria, sickle cell disease, thrombotic thrombocytopenic purpura, following organ transplantation, human immunodeficiency virus infection, intravenous globulin therapy, chemotherapy, or vascular endothelial injury by some investigators $[2,3]$.

In healthy subjects, cerebral autoregulation is maintained by both myogenic and neurogenic components, the latter being dependent on the degree of sympathetic innervation. In patients with PRES, passive overdistension of the blood vessels due to rise in BP blunts the myogenic response. The neurogenic component thus predominates and there is breakdown of the blood-brain barrier with focal transudation of fluid and petechial hemorrhages. The predilection for involvement of posterior circulation territories is generally accepted to result from the relatively sparse sympathetic innervation of the vertebrobasilar circulation [4].

Imaging is essential for the differential diagnosis of PRES, which includes stroke, cerebral venous thrombosis, and encephalitis. Neuroimaging shows confluent, usually symmetric, subcortical white matter lesions, which can extend to the cortical surface and into the deep white matter preferentially involving the parietooccipital regions of the 
brain. Structures such as brain stem, basal ganglia, cerebellum and frontal lobes can be affected as well. Localized mass effect and subtle enhancement within the lesions have been described, but are not seen consistently. DWI and apparent diffusion coefficient (ADC) maps can help in differentiating PRES from ischemic events by showing an increased ADC consistent with vasogenic edema rather than restricted diffusion which is typical of acute ischemic damage [5]. The extent of T2 and DWI signal intensity correlates well with patient outcome and can help guide more aggressive treatment in more severely affected patients. Atypical MRI findings such as infarction with permanent residual lesions and hemorrhage are becoming increasingly reported [6].

We describe a case with clinical and imaging findings consistent with PRES that occurred after spinal surgery without any of the described risk factors. The rapid increase in BP in our patient in the postoperative period might have contributed to this syndrome. Though isolated reports of PRES following surgery are available in literature, it has mostly been in the background of disorders otherwise known to manifest with hypertension $[7,8]$. None have presented following spine surgery. Dorsal cord injury following trauma has been known to cause sympathetic dysreflexia, which in turn can cause systemic hypertension. Our observation in this case implicates cord compression followed by sympathetic dysfunction as the probable mechanism for onset of PRES. The other possibility that a general anesthetic agent can trigger PRES needs to be considered.

In conclusion, acute spinal cord compression must be included as one of the possible etiologies for PRES. Early recognition of this clinico-radiologic entity in the postoperative period can prevent mortality by prompt treatment.

\section{REFERENCES}

1. Hinchey J, Chaves C, Appignani B, et al. A reversible posterior leukoencephalopathy syndrome. N Engl J Med 1996;334:494-500

2. Gümüs H, Per H, Kumandas S, Yikilmaz A. Reversible posterior leukoencephalopathy syndrome in childhood: report of nine cases and review of the literature. Neurol Sci 2010;31:125-31.

3. Lamy C, Oppenheim C, Méder JF, Mas JL. Neuroimaging in posterior reversible encephalopathy syndrome. J Neuroimaging 2004;14:89-96.

4. Schwartz RB, Feske SK, Polak JF, et al. Preeclampsiaeclampsia: clinical and neuroradiographic correlates and insights into the pathogenesis of hypertensive encephalopathy. Radiology 2000;217:371-6.

5. Covarrubias DJ, Luetmer PH, Campeau NG. Posterior reversible encephalopathy syndrome: prognostic utility of quantitative diffusion-weighted MR images. AJNR Am J Neuroradiol 2002;23:1038-48.

6. Aranas RM, Prabhakaran S, Lee VH. Posterior reversible encephalopathy syndrome associated with hemorrhage. Neurocrit Care 2009;10:306-12.

7. Rangi PS, Partridge WJ, Newlands ES, Waldman AD. Posterior reversible encephalopathy syndrome: a possible late interaction between cytotoxic agents and general anaesthesia. Neuroradiology 2005;47:586-90.

8. Triquenot-Bagan A, Gerardin E, Guegan-Massardier E, Onnient Y, Leroy F, Mihout B. Postoperative reversible posterior leukoencephalopathy syndrome. Cerebrovasc Dis 2003;16:430-2. 